\title{
Microplane constitutive model for porous isotropic rocks
}

\author{
Zdeněk P. Bažant*, ${ }^{*}$ and Goangseup Zi \\ Department of Civil Engineering, Northwestern University, Evanston, IL 60208, USA
}

\begin{abstract}
SUMMARY
The paper deals with constitutive modelling of contiguous rock located between rock joints. A fully explicit kinematically constrained microplane-type constitutive model for hardening and softening non-linear triaxial behaviour of isotropic porous rock is developed. The microplane framework, in which the constitutive relation is expressed in terms of stress and strain vectors rather than tensors, makes it possible to model various microstructural physical mechanisms associated with oriented internal surfaces, such as cracking, slip, friction and splitting of a particular orientation. Formulation of the constitutive relation is facilitated by the fact that it is decoupled from the tensorial invariance restrictions, which are satisfied automatically. In its basic features, the present model is similar to the recently developed microplane model M4 for concrete, but there are significant improvements and modifications. They include a realistic simulation of (1) the effects of pore collapse on the volume changes during triaxial loading and on the reduction of frictional strength, (2) recovery of frictional strength during shearing, and (3) the shearenhanced compaction in triaxial tests, manifested by a deviation from the hydrostatic stress-strain curve. The model is calibrated by optimal fitting of extensive triaxial test data for Salem limestone, and good fits are demonstrated. Although these data do not cover the entire range of behaviour, credence in broad capabilities of the model is lend by its similarity to model M4 for concrete- an artificial rock. The model is intended for large explicit finite-element programs. Copyright (C) 2002 John Wiley \& Sons, Ltd.
\end{abstract}

KEY WORDS: microplane model; porous rock; plasticity; fracturing; material modelling; finite elements

\section{INTRODUCTION}

Development of material models for the analysis of deformation and failure of rock is a complex problem which has been studied for a long time. Significant advances have been achieved [1-6] but a realistic and versatile model of broad applicability, suitable for large-scale numerical computations, is still unavailable.

Two kinds of material models may be distinguished: (a) The models for the behaviour of large rock masses, whose inelastic behaviour is totally dominated by deformations at the rock joints, and (b) the models for the behaviour of contiguous rock between the joints. For problems of geotechnical engineering, the former are usually much more important, but for some specific applications contemplated here, such as the impact and penetration of missiles through rock, or

\footnotetext{
*Correspondence to: Z. P. Bažant, Department of Civil Engineering, Northwestern University, Evanston, IL 60208, USA

†E-mail: z-bazant@northwestern.edu
}

Contract/grant sponsor: U.S. Army Engineer Waterways Experiment Station (WES); contract/grant number: DAC39-94-C-0025

Copyright (C) 2002 John Wiley \& Sons, Ltd.

Received 13 February 2002 Revised 23 August 2002 
the effects of groundshock and blast, as well as anchor insertion, fragmentation and drilling, the latter are essential. This study deals exclusively with the latter.

Among the studies of contiguous rock located between the joints, two types of models may again be distinguished: (a) micromechanics of various physical mechanisms of inelastic deformation in the microstructure [2,3], and (b) the development of constitutive and fracture models on the macroscopic continuum level [4-8]. The objective of this study is to develop for isotropic porous rocks a model that is basically of the latter type but incorporates an approximate characterization of oriented physical mechanisms in the microstructure, i.e. which has an ingredient of the former type.

The porous brittle rocks are characterized by brittle-ductile transition in triaxial loading. Such rocks fail by brittle faulting at low pressures, but become ductile and gradually harden at high pressures. When deviatoric stresses accompany high pressure, an additional volume change called the shear-enhanced compaction takes place. Fracture mechanics may be regarded as the limiting case of constitutive models with strain-softening damage, but lies outside the scope of the present study.

The best existing macroscopic constitutive models formulated in terms of the stress and strain tensors and their invariants are based on irreversible thermodynamics and internal variables. Such models perform well in computations. However, they are basically phenomenological, and thus not sufficiently realistic for some demanding applications. As has been pointed out [5], their parameters are not clearly connected to physical mechanisms, especially to those of an oriented character. The models based on continuum damage mechanics employ the crack density tensor which characterizes the global damage due to cracks of all orientations. This works well for the overall effective stiffness only as long as the stiffness is not reduced too much, but is unrealistic for predicting failure since the failure typically depends on the growth of cracks of one dominant orientation [4] or frictional-dilatant slip in a particular direction.

\section{EVOLUTION AND BASIC FEATURES OF MICROPLANE MODEL}

The evolution of the microplane modelling approach can be traced back to a pioneering idea of G.I. Taylor [9], who proposed characterizing the constitutive behaviour of polycrystalline metals by relations between the stress and strain vectors acting on planes of various possible orientations within the material (later named the microplanes) and determining the macroscopic strain and stress tensors as a summation of all these vectors under the assumption that either the stress or the strain vector acting on each of these planes is the projection of the stress or strain tensor (this assumption is called the static or kinematic constraint of the microplanes). Batdorf and Budiansky [10] were the first to put Taylor's idea into practice. They developed a realistic model with static constraint for plasticity of polycrystalline metals based on plastic slip on the crystallographic planes, still considered among the best. Many other researchers subsequently extended or modified this approach to metals [11-16]. Extensions of this approach for the hardening inelastic response of soils and rocks were also made [17-20].

To adapt Taylor's idea to strain-softening behaviour of concrete due to distributed cracking, Bažant [21] and Bažant and Oh [22] noted that a kinematic constraint was necessary for reasons of stability. They took into account the fracturing normal strains on the microplanes, and introduced the principle of virtual work as a means of obtaining the strain tensor from the microplane stress components. To apply a strain-softening microplane model in finite-element 
programs, they adopted the crack-band model [23,24], in which the strain softening is related to the fracture energy of the material and the effective size of the fracture process zone (however, since the post-peak slope in References [21,22] was not adjustable, the full crack-band model could not be used and the element size had to be fixed). From that time on, further developments of Taylor's idea for plastic (non-softening) materials and for softening quasibrittle materials have proceeded along separate, rather different, lines (in detail, see the review of Brocca and Bažant [25]).

To develop a microplane model for general non-linear triaxial behaviour of concrete, a volumetric-deviatoric split on the microplanes is inevitable. It was introduced in Reference [26], although a thermodynamically consistent energetic treatment of this split appeared much later [27]. Formulating a purely geometric microplane damage tensor, Carol and Bažant [28] advanced a microplane formulation fitting the framework of continuum damage mechanics. Carol et al. [29] showed that the microplane model logically ensues from the general framework of continuum thermodynamics if one assumes that the free energy density is simply a sum of the partial free energy densities associated with microplanes of all spatial orientations.

Despite conceptual simplicity of the microplane model, its formulation and calibration for a broad set of experimental data is a challenging problem. Gradual progress has come in a series of progressively improved microplane models for concrete (in retrospect named M1, M2, M3 and M4 [26, 27, 30-33]; for a detailed account of the history of microplane model, see mainly References [27,34-36]). This progress has been brought about chiefly by approximate simulation of various distinct physical sources of oriented inelastic behaviour, such as tensile microcracking, slip, friction, lateral confinement, splitting and lateral spreading due to compression, and the collapse and closing of pores. In contrast to the classical tensorial constitutive models, the microplane model is particularly suited for this purpose, thanks to employing no tensors but stress and strain vectors on planes of various orientations. This is one important advantage, lending the model conceptual simplicity. For example, a relation between the first and second invariants of the stress tensor, as used in the Drucker-Prager yield surface [36], is only a vague overall characterization of internal friction in a material. In reality, the frictional slip occurs on planes of a certain particular orientation, and friction is properly a relation between the shear and normal stress components on each slip plane. The microplane model can capture it easily (in this respect, though, one must admit that, in a kinematically constrained model, the stresses acting on a given plane are not the actual stresses but only their approximations).

Model M4, the latest microplane model for concrete, has been used in EPIC [37], an explicit finite-strain hydro-code (wave-code), to analyse missile impact and penetration, blast and groundshock, in simulations involving up to several million finite elements, with the microplane model used in 40000 elements. With the most powerful computers that exist today, the excess of computational work of microplane model over the classical tensorial constitutive models is no longer a problem. This is especially true for large implicit finite-element systems for which the computer time is dominated by the size of the stiffness matrix rather than the constitutive model. However, the difficulty of obtaining a tangential stiffness matrix precludes benefiting from efficient implicit methods.

Aside from the advantages already mentioned, another one is the automatic simulation of the so-called vertex effect, i.e. the fact that the response to a loading whose direction in the stress space is tangent to the current effective yield surface (loading 'to the side') is not elastic, as predicted by all the classical tensorial models applied in practice, but inelastic and, in fact as 
recently demonstrated experimentally, much softer than elastic [38]. The capability to simulate this phenomenon can be understood if one regards the microplane model as a multisurface plasticity model, in which each of the microplanes (whose number must be at least 21) corresponds to several independent yield surfaces. When the loading direction in the ninedimensional stress space changes abruptly, the currently active yield surfaces are deactivated but other yield surfaces get immediately activated, thus causing the tangential stiffness for loading to the side to become smaller than the elastic one.

A further advantage of the microplane approach is the automatic representation of crosseffects between different orientations. For example, if the tensile strength is less than the compressive strength, as is typical of concrete as well as rock, then a non-dilatant shearing on one microplane nevertheless automatically produces volume dilatancy overall, by the virtue of the fact that the kinematic constraint induces normal stresses on inclined microplanes and that the compressive stresses produced by shearing on microplanes inclined by about $45^{\circ}$ are larger in magnitude than the tensile stresses produced on microplanes inclined by about $-45^{\circ}$. Still another advantage is that the kinematically constrained microplane model also spontaneously simulates fatigue because it leads to a gradual build-up of residual self-equilibrated stresses in the microplane system during load cycles [39]. Furthermore, despite path independence of the microplane constitutive law, a complex path dependence is automatically generated by numerous possible combinations of loading and unloading on various microplanes. The microplane constitutive law also allows easy consideration of strain-softening yield limits (as functions of strain components), which would be prohibitively complex on the tensorial level.

It is also convenient that, on the microplane level, the transition from elastic behaviour to yielding can be abrupt even if the material exhibits gradual hardening on the macroscale. The reason is that microplanes of different orientations enter yielding at different stages of the loading process.

With a view toward future generalizations to anisotropic rocks, it should be noted that with the microplane model it would be easy to model the orthotropy exhibited by some rocks. It would suffice to introduce on each microplane orientation-dependent material parameters or weight function.

In parallel with the present study, the physical basis of model M4 has been deepened (in model M5 [40]) by relating the softening behaviour to the fracture energy of the material and to the characteristic material length representing the effective size of the fracture process zone. Introducing these fracture energy aspects into a model for rock is left for future work.

Aside from concrete, microplane models have been developed at Northwestern University for clay [41], soils [42], steel [34], shape-memory alloys [43] and polymeric foams [44]. In what follows, a microplane model for isotropic porous rock will be developed, with model M4 for concrete used as the starting point. First, the basic formulation of microplane model needs to be briefly reviewed.

\section{REVIEW OF BASIC RELATIONS OF MICROPLANE MODEL}

\subsection{Formulation of kinematic constraint}

The kinematic constraint, which ensures stability of the material model in the case of strain softening [21], means that the strain vector on each microplane (Figure 1) is the resolved 


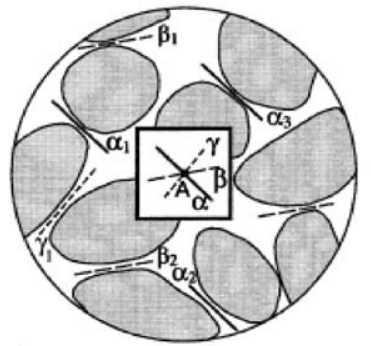

(a)

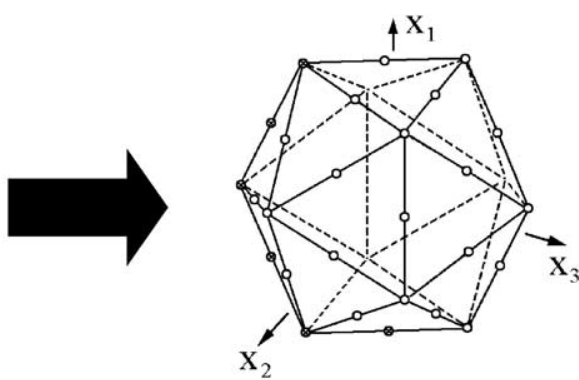

(b)

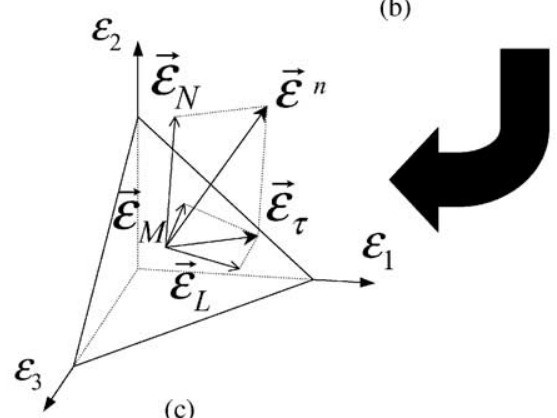

(c)

Figure 1. Kinematically constrained microplane model: (a) microstructure and microplanes in a representative volume of a cohesive granular material; (b) icosahedron where the circled points represent the directions of microplane normals in a 21-point optimal Gaussian integration formula [45]; and (c) strain projections on each microplane (kinematical constraint).

component of the macroscopic strain tensor $\varepsilon_{i j}$. The components of the strain vector $\varepsilon_{j}^{n}$ on any microplane are $\varepsilon_{j}^{n}=\varepsilon_{j k} n_{k}$ where $n_{k}$ is the unit normal of the microplane (Figure 1c) [26]. The normal strain vector and its magnitude are

$$
\begin{gathered}
\varepsilon_{N_{i}}=n_{i} n_{j} n_{k} \varepsilon_{j k} \\
\varepsilon_{N}=n_{j} \varepsilon_{j}^{n}=n_{j} n_{k} \varepsilon_{j k}=N_{i j} \varepsilon_{i j}
\end{gathered}
$$

where $N_{i j}=n_{i} n_{j}$, and the repetition of Latin lowercase subscripts indicates summation over $1,2,3$. The shear strain vector (Figure 1c) is

$$
\begin{aligned}
\varepsilon_{T_{i}} & =\varepsilon_{i}^{n}-\varepsilon_{N_{i}} \\
& =\left(\delta_{i j}-n_{i} n_{j}\right) n_{k} \varepsilon_{j k}=\frac{1}{2}\left(n_{j} \delta_{i k}+n_{k} \delta_{i j}-2 n_{i} n_{j} n_{k}\right) \varepsilon_{j k}
\end{aligned}
$$

where $\delta_{i j}$ is Kronecker's unit delta tensor. Because the shear stress and strain directions generally do not coincide, the shear strain is further decomposed into two orthogonal components $\varepsilon_{M}$ and $\varepsilon_{L}$ in directions, $m_{i}$ and $l_{i}$. To minimize directional bias in a simple way, the directions $m_{i}$ and $l_{i}$ on the individual microplanes are alternatively chosen to be normal to axes $x_{1}-x_{3}$. If $m_{i}$ is chosen normal to $x_{3}$,

$$
m_{1}=n_{2}\left(n_{1}^{2}+n_{2}^{2}\right)^{-1 / 2}, \quad m_{2}=-n_{1}\left(n_{1}^{2}+n_{2}^{2}\right)^{-1 / 2}, \quad m_{3}=0
$$

$l_{i}$ is obtained by the vector product of $m_{i}$ and $n_{i}$, i.e. $l_{i}=e_{i j k} m_{j} n_{k}$, where $e_{i j k}$ is the permutation symbol (which is 1 for even permutation subscripts, -1 for odd permutation, and 0 if any two 
indices are the same). The shear strain components in the directions of $m_{i}$ and $l_{i}$ are $\varepsilon_{M}=$ $m_{i}\left(\varepsilon_{i j} n_{j}\right)$ and $\varepsilon_{L}=l_{i}\left(\varepsilon_{i j} n_{j}\right)$, and because of the requirement of symmetry,

$$
\varepsilon_{M}=M_{i j} \varepsilon_{i j}, \quad \varepsilon_{L}=L_{i j} \varepsilon_{i j}
$$

where $M_{i j}=\left(m_{i} n_{j}+m_{j} n_{i}\right) / 2$ and $L_{i j}=\left(l_{i} n_{j}+l_{j} n_{i}\right) / 2[26,30]$.

\subsection{Static equivalence}

Because of the kinematic constraint, static equivalence of the continuum stress tensor $\sigma_{i j}$ and the microplane stress components $\sigma_{N}, \sigma_{L}, \sigma_{M}$ can be enforced only approximately. This is done by the virtual work theorem, written for a unit hemisphere [21] as

$$
\begin{aligned}
\frac{2 \pi}{3} \sigma_{i j} \delta \varepsilon_{i j} & =\int_{\Omega}\left(\sigma_{N} \delta \varepsilon_{N}+\sigma_{L} \delta \varepsilon_{L}+\sigma_{M} \delta \varepsilon_{M}\right) \mathrm{d} \Omega \\
& =\int_{\Omega}\left(\sigma_{N} N_{i j}+\sigma_{L} L_{i j}+\sigma_{M} M_{i j}\right) \delta \varepsilon_{i j} \mathrm{~d} \Omega
\end{aligned}
$$

The normal stress depends on not only the normal strain but also the lateral deformation. The lateral deformation of a microplane is measured by the spreading strain $\varepsilon_{\mathrm{S}}$, which is the average of the normal strains in all the directions parallel to the microplane. If $\varepsilon_{\mathrm{D}}=\varepsilon_{N}-\varepsilon_{\mathrm{V}}=$ deviatoric strain, with $\varepsilon_{\mathrm{V}}=\varepsilon_{k k} / 3=$ volumetric strain, the volume change may be written as

$$
3 \varepsilon_{\mathrm{V}}=3\left(\varepsilon_{N}-\varepsilon_{\mathrm{D}}\right)=\varepsilon_{N}+2 \varepsilon_{\mathrm{S}}
$$

which means that $\varepsilon_{\mathrm{S}}=\left(3 \varepsilon_{\mathrm{V}}-\varepsilon_{N}\right) / 2$. Because $\varepsilon_{\mathrm{V}}$ is a three-dimensional invariant, $\varepsilon_{\mathrm{V}}$ is more convenient to use than $\varepsilon_{\mathrm{s}}$. The normal stress on each microplane is also split into its volumetric and deviatoric parts, $\sigma_{\mathrm{V}}$ and $\sigma_{\mathrm{D}}$, and then the variational equation (7), written separately for $\sigma_{\mathrm{V}} \delta \varepsilon_{\mathrm{V}}$ and $\sigma_{i j}^{\mathrm{D}} \delta \varepsilon_{i j}^{\mathrm{D}}$ where $\sigma_{i j}^{\mathrm{D}}=\sigma_{i j}-\delta_{i j} \sigma_{\mathrm{V}}$, reads $\varepsilon_{i j}^{\mathrm{D}}=\varepsilon_{i j}-\delta_{i j} \varepsilon_{\mathrm{V}}$

$$
\begin{gathered}
\sigma_{i j}=\sigma_{\mathrm{V}} \delta_{i j}+\sigma_{i j}^{\mathrm{D}} \\
\sigma_{i j}^{\mathrm{D}}=\frac{3}{2 \pi} \int_{\Omega}\left[\sigma_{\mathrm{D}}\left(N_{i j}-\frac{\delta_{i j}}{3}\right)+\sigma_{L} L_{i j}+\sigma_{M} M_{i j}\right] \mathrm{d} \Omega
\end{gathered}
$$

With this volumetric-deviatoric split, it is possible to reproduce the full range of Poisson's ratio $-1 \leqslant v \leqslant 0.5$ of elastic deformations. The term $-\delta_{i j} / 3$ in (10) ensures that $\sigma_{k k}^{\mathrm{D}}=0$ even when $\int_{\Omega} \sigma_{\mathrm{D}} \mathrm{d} \Omega \neq 0$. The integration is conducted numerically by optimal Gaussian quadrature on a hemispherical surface [22]

$$
\sigma_{i j}=\frac{3}{2 \pi} s_{i j} \approx 6 \sum_{\mu=1}^{N_{m}} w_{\mu} s_{i j}^{(\mu)}
$$

where $s_{i j}=\int_{\Omega}\left(\sigma_{N} N_{i j}+\sigma_{L} L_{i j}+\sigma_{M} M_{i j}\right) \mathrm{d} \Omega$ and $N_{m}$ is the number of all microplanes.

\section{MICROPLANE MODELLING OF INELASTIC BEHAVIOUR}

The behaviour of quasibrittle materials under uniaxial, biaxial, triaxial loading conditions exhibits several characteristic features, such as pore collapse and vertex effect, which are extremely complicated and hardly describable with a tensorial constitutive model. 
The inelastic behaviours of concrete are described by the introduction of the so-called stressstrain boundaries $[31,32]$. Inside the boundaries, the stresses change only by linear elasticity, i.e.

$$
\Delta \sigma_{\mathrm{V}}=E_{\mathrm{V}} \Delta \varepsilon_{\mathrm{V}}, \quad \Delta \sigma_{\mathrm{D}}=E_{\mathrm{D}} \Delta \varepsilon_{\mathrm{D}} \quad \text { and } \quad \Delta \sigma_{T}=E_{T} \Delta \varepsilon_{T}
$$

where, for brevity, $\Delta \sigma_{T}$ and $\Delta \varepsilon_{T}$ stand for either $\Delta \sigma_{M}$ and $\Delta \varepsilon_{M}$, or $\Delta \sigma_{L}$ and $\Delta \varepsilon_{L} ; E_{\mathrm{V}}=$ $E /(1-2 v), E_{\mathrm{D}}=5 E /(2+3 \mu)(1+v), E_{T}=\mu E_{\mathrm{D}}, E$ is Young's modulus (macroscopic), and $\mu$ is a parameter that can be chosen. The best choice (for which a purely geometric damage tensor exists) is $\mu=1[28,31,46]$. If the stress value exceeds the associated boundary, the stress is dropped at constant strain to the value for the boundary. Despite the abrupt drop of the stress magnitude, a smooth overall behaviour is achieved by the interaction of many microplanes, and by taking small loading steps. All the boundaries, except for the compressive volumetric boundary and the shear boundary, undergo strain softening. The strain softening physically represents the fracturing process in a smeared manner. After multiplication by the crack band width (characteristic length), the area under the microplane stress-strain diagram delimited by the strain axis, by the elastic unloading path emanating from the peak, and by the softening boundary has the meaning of the fracture energy on the microplane level.

Using semi-empirical considerations coupled with physical concepts, we will now set up the microplane constitutive model for rock, which will be labelled as M4R, since it represents an adaptation and refinement of model M4 for concrete [27,33]. The inelastic behaviour is characterized by strain-dependent yield limits, called the stress-strain boundaries, which we will define in dimensionless variables. To describe the boundaries, we will need a total of 21 nonadjustable parameters, expected to be common to various rocks. The differences among individual rocks are taken into account by parameters $k_{1}-k_{4}$, which are easily adjustable; $k_{1}$ controls scaling in radial direction in the stress-strain plane (while the vertical scaling is controlled by $E$, and the horizontal scaling is controlled by $k_{1} / E$ ); parameter $k_{2}$ controls the frictional strength; and parameters $k_{3}$ and $k_{4}$ characterize the compressive hydrostatic stress-strain curve.

\subsection{Horizontal segments of boundaries: plastic limits}

The experimental data for uniaxial tension or compression of quasibrittle materials display smooth rounded peaks, which suggests the existence of a limited ductility. To capture this feature, most boundaries except the compressive volumetric boundary and the shear boundary, begin by short horizontal segments (corresponding to constant strength limits). These horizontal segments represent microplane yield limits. The length of these segments controls the roundness of response curves near the peaks and of the hysteretic loops under cyclic loading.

\subsection{Normal stress-strain boundary}

The normal stress component is the sum of the volumetric stress $\sigma_{\mathrm{V}}$ and the deviatoric stress $\sigma_{\mathrm{D}}$, both of which are limited by strain-softening boundaries [31]. Now note that when there are two simultaneous softening processes, the softening will normally localize into one of them [47-49]. This may cause $\varepsilon_{\mathrm{D}}$ and $\varepsilon_{\mathrm{V}}$ to have large values of opposite signs, producing excessively large $\sigma_{N}$. For this reason, it is convenient to impose also a separate softening tensile boundary on the total normal stress. This boundary approaches zero at infinite normal strain and its descent becomes more rapid as the confinement pressure increases. Physically, the tensile normal boundary characterizes the tensile cracking. It is given by 


$$
\sigma_{N}^{b+}=E k_{1} c_{1} \exp \left(-\frac{\left\langle\varepsilon_{N}-k_{1} c_{1} c_{2}\right\rangle}{k_{1} c_{3}+\left\langle-c_{4} \sigma_{\mathrm{V}} / E_{\mathrm{V}}\right\rangle}\right)
$$

The Macaulay brackets, defined as $\langle x\rangle=\operatorname{Max}(x, 0)$, are used here and in the subsequent formulas as a simple way to define the horizontal segments of the boundaries. The value of $\sigma_{\mathrm{V}}$ needs to be here taken from the previous load step or the previous iteration of the current step.

\subsection{Deviatoric stress-strain boundaries}

The deviatoric boundaries control the axial crushing strains with lateral expansion in compression when the lateral confinement is too weak. Most quasibrittle materials expand when subjected to unconfined compression. Such expansion is normally obtained by interaction (due to kinematic constraint) with microplanes inclined by about $\pm 45^{\circ}$ (Figure 2) [50], provided that the compression strength is higher than the tensile strength, i.e. $-\sigma_{\mathrm{D}}^{b-}\left(-\varepsilon_{\mathrm{D}}\right)>\sigma_{\mathrm{D}}^{b+}\left(\varepsilon_{\mathrm{D}}\right)$. The compressive and tensile deviatoric boundaries are defined as

$$
\sigma_{\mathrm{D}}^{b-}=-\frac{E_{\mathrm{D}} k_{1} c_{8}}{1+\left(\left\langle-\varepsilon_{\mathrm{D}}-k_{1} c_{8} c_{9}\right\rangle / k_{1} c_{7}\right)^{2}} \quad \text { and } \quad \sigma_{\mathrm{D}}^{b+}=\frac{E_{\mathrm{D}} k_{1} c_{5}}{1+\left(\left\langle\varepsilon_{\mathrm{D}}-k_{1} c_{5} c_{6}\right\rangle / k_{1} c_{20}\right)^{2}}
$$

where they are scaled with respect to the deviatoric modulus $E_{\mathrm{D}}$ rather than $E$. Because the deviatoric boundaries represent the failure surface under pure deviatoric deformation, it is more natural to choose $E_{\mathrm{D}}$ as the scaling factor. By the same reason, the shear boundary is scaled by $E_{T}$, and the volumetric boundaries by $E_{\mathrm{V}}$.

\subsection{Frictional yield surface}

The shear boundary models friction, which depends on stress non-linearly. The frictional yield strength is proportional to the normal stress which is acting on the frictional plane. The friction angle, which is defined as the ratio of the frictional shear strength to the normal stress across the plane of sliding, is relatively large at low normal stresses (about $40^{\circ}$ ) and becomes smaller as the compressive stress magnitude increases. When the normal stress is zero, the frictional shear strength is not zero, which means that there is finite cohesion. These features were captured in

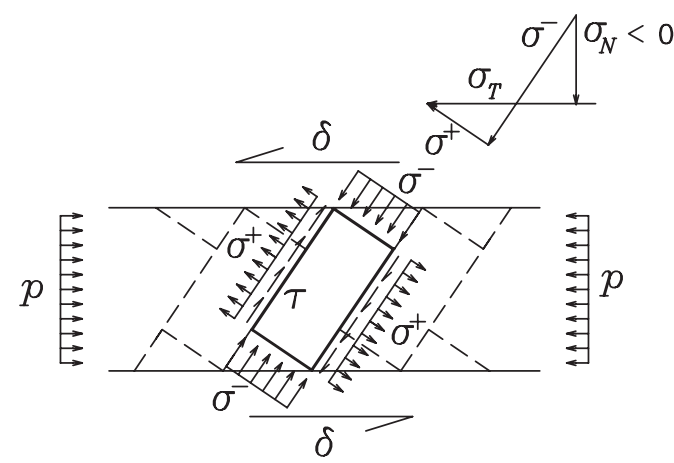

Figure 2. Difference in magnitudes of inclined compressive and tensile stresses caused by horizontal shear at constant vertical strain (this difference causes vertical normal stress, and if this stress is not resisted, then dilatancy). 
M4 by means of a yield surface in the space of shear and normal stresses having the form of a hyperbola intersecting the normal stress axis at a finite value $\sigma_{N}^{0}$. The cohesion depends on volumetric confinement. It will be convenient to describe first the boundaries used in M4 for concrete, which are

$$
\begin{gathered}
\sigma_{T}^{\mathrm{M} 4}=\frac{E_{T} k_{1} k_{2} c_{10}\left\langle\sigma_{N}^{0}-\sigma_{N}\right\rangle}{E_{T} k_{1} k_{2}+c_{10}\left\langle\sigma_{N}^{0}-\sigma_{N}\right\rangle} \\
\sigma_{N}^{0}=\frac{E_{T} k_{1} c_{11}}{\left(1+c_{12} \frac{\left\langle\varepsilon_{\mathrm{V}}\right\rangle}{k_{1}}\right)}
\end{gathered}
$$

where $\sigma_{T}$ means the shear stress components, either $\sigma_{L}$ or $\sigma_{M}$. The latter expression can be adopted for rock but the former will have to be modified; $E_{T} k_{1} k_{2}=$ ultimate frictional shear strength when $-\sigma_{N} \rightarrow \infty$. Although no softening is explicitly given by (15), the macroscopic shear stress-strain curve does exhibit post-peak descent due to the interaction with the normal stress, whose softening behaviour is described by (16). The higher the normal stress, the higher is the frictional strength. When the normal stress softens, the frictional strength decreases, too.

An important purpose of the frictional shear boundary is to capture the brittle-ductile transition observed in triaxial tests of concrete. Aside from axial splitting, the failure of brittle materials under compression is caused by shear. The failure mechanism is sketched in Figure 2. Because the compressive strength is higher than the tensile strength, the brittle failure is caused by softening of $\sigma^{+}$, when the confining pressure $p$ is very small $\left(p=-\sigma_{\mathrm{V}}\right)$. As $p$ increases, $\sigma^{+}$ decreases and becomes negative. Because the shear strength is influenced by $\sigma_{N}$ which depends on $\sigma^{+}$through yield surface (16), it also increases, due to the increase of pressure $p$. In such a condition, the deformation is mainly resisted by the shear stress, for which the shear strength is determined by (16). This makes the failure ductile.

Model M4 successfully captures the brittle-ductile transition in triaxial tests of concrete [33]. However it is found that M4 gives excessive lateral contraction at the onset of triaxial loading (Figure 3), which is not always unrealistic because either a contractive or an expansive deviation of lateral strain from the hydrostatic response can occur [51].

The standard triaxial test consists of two loading stages: (1) The initial loading is by hydrostatic pressure until the desired confinement pressure is reached, and then (2) the axial compression is increased further while the confinement pressure is kept constant. In the second stage, the material undergoes the first deviatoric deformation (Figure 4), which must initially be elastic. This initial deviatoric deformation causes the aforementioned excessive lateral contraction as long as the elastic deviatoric tangent modulus remains constant. Of course, this excessive contraction vanishes as the slope of the hydrostatic response (point 3 in Figure 4a) increases. The abrupt slope change of the hydrostatic response also contributes to the excessive lateral contraction, which will be discussed later.

To correct this problem, one can make the inelastic deviatoric tangent modulus depend on the confining pressure. The macroscopic deviatoric tangent modulus is the superposition from all microplanes of the slopes $\mathrm{d} \sigma_{\mathrm{D}} / \mathrm{d} \varepsilon_{\mathrm{D}}$ and $\mathrm{d} \sigma_{T} / \mathrm{d} \varepsilon_{T}$ (along the loading path) [46]. Because the deviatoric strain is very small at the onset of triaxial loading, $\mathrm{d} \sigma_{\mathrm{D}} / \mathrm{d} \varepsilon_{\mathrm{D}}$ is equal to $E_{\mathrm{D}}$, which is constant. Because $\mathrm{d} \sigma_{T} / \mathrm{d} \varepsilon_{T}$ is controlled by the hyperbolic frictional boundary (16), it appears that, if the frictional strength is small enough, $\mathrm{d} \sigma_{T} / \mathrm{d} \varepsilon_{T}$ becomes less than $E_{T}$. This observation 


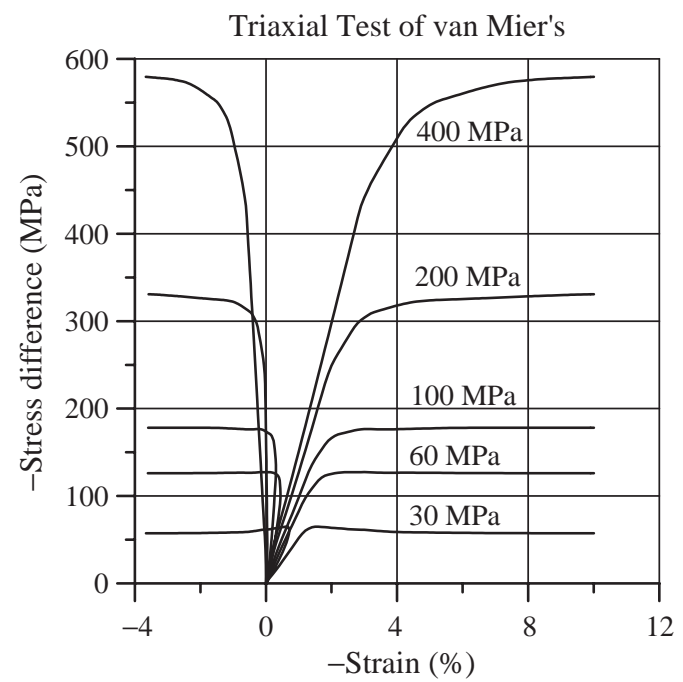

Figure 3. Triaxial test simulation by M4.
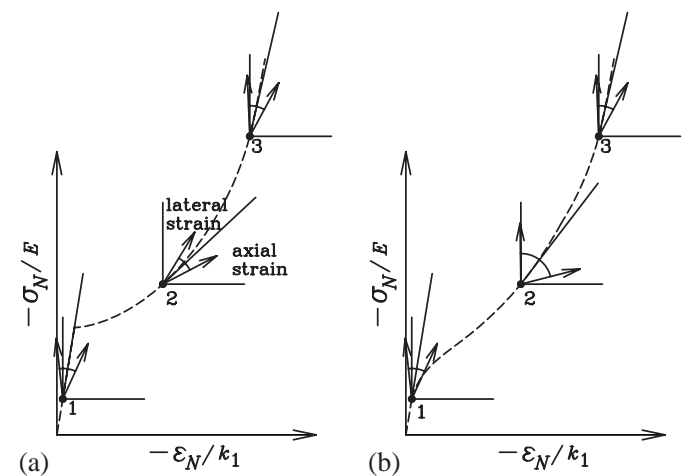

Figure 4. The direction of axial and lateral strain at the onset of triaxial loading after hydrostatic confinement; (a) with incremental elastic deviatoric stiffness and abrupt change of hydrostatic curve, and (b) with inelastic deviatoric stiffness and a smoothed hydrostatic curve.

indicates the simplest way to obtain the inelastic deviatoric tangent modulus. Physically, this reduction of the frictional strength may be attributed to pore collapse.

As will be discussed later in the modelling of volumetric boundary, the aforementioned compaction, which may be called the shear-enhanced compaction, represents an additional volume change due to shearing (distortion). A physical mechanism that could explain this observed behaviour is the densification due to particle interlocking induced by shear, which is absent at hydrostatic volume compaction. The strength reduction is gradually recovered by interlocking of adjacent particles during further deformation. The maximum shear strain on each microplane is chosen to characterize this recovery.

As a result of the foregoing discussion, two new functions describing the shear resistance reduction and its recovery are proposed. 


$$
\sigma_{T}^{b}=\sigma_{T}^{\mathrm{M} 4} f_{r}\left(\left|\varepsilon_{T}\right|^{\max }\right)
$$

where

$$
\begin{gathered}
f_{r}\left(\left|\varepsilon_{T}\right|^{\max }\right)=(1-\varphi)\left[1-\exp \left(-c_{25}\left|\varepsilon_{T}\right|^{\max } / k_{1}\right)\right]+\varphi \\
\varphi\left(\sigma_{\mathrm{V}}^{\min }\right)=c_{22}+\frac{1-c_{22}}{1+\exp \left[c_{23}\left(c_{18}-3 \sigma_{\mathrm{V}}^{\min } / E_{\mathrm{V}} k_{1}\right)\right]}
\end{gathered}
$$

in which $\varphi\left(\sigma_{\mathrm{V}}^{\min }\right)=$ factor of strength reduction due to pore collapse (Figure 5(a)) and $f_{r}\left(\left|\varepsilon_{T}\right|^{\max }\right)=$ factor of strength recovery at large shear deformation (Figure 5(b)). Here, $3 \sigma_{\mathrm{V}}^{\min } / E_{\mathrm{V}}$ can be seen as an equivalent hydrostatic strain for the strength reduction due to pore collapse; $\left|\varepsilon_{T}\right|^{\max }$ and $\sigma_{\mathrm{V}}^{\min }$ are the maximum and minimum of $\varepsilon_{M}$ or $\varepsilon_{L}$, and of $\sigma_{\mathrm{V}}$, attained so far; they need to be stored in the process of computation. The shear boundary Equation (17) is plotted in Figure 6(a).

\subsection{Volumetric boundaries}

Porous rocks as well as concretes exhibit no softening under increasing hydrostatic pressure; rather, after an initial pore collapse they progressively harden due to closure of pores. Thus, the slope of the hydrostatic stress-strain curve of porous geomaterials first dramatically decreases, just after pore collapse (point 2 in Figure 4(a)) [33,52], and then gradually increases. This hardening may be described by an exponential-type boundary in M4 [27]:

$$
\sigma_{\mathrm{V}}^{b-\mathrm{M} 4}=E_{\mathrm{V}} k_{1} k_{3} \exp \left(-3 \varepsilon_{\mathrm{V}} / k_{1} k_{4}\right)
$$

where the strength and the slope are controlled, respectively, by $k_{3}$ and $k_{4}$.
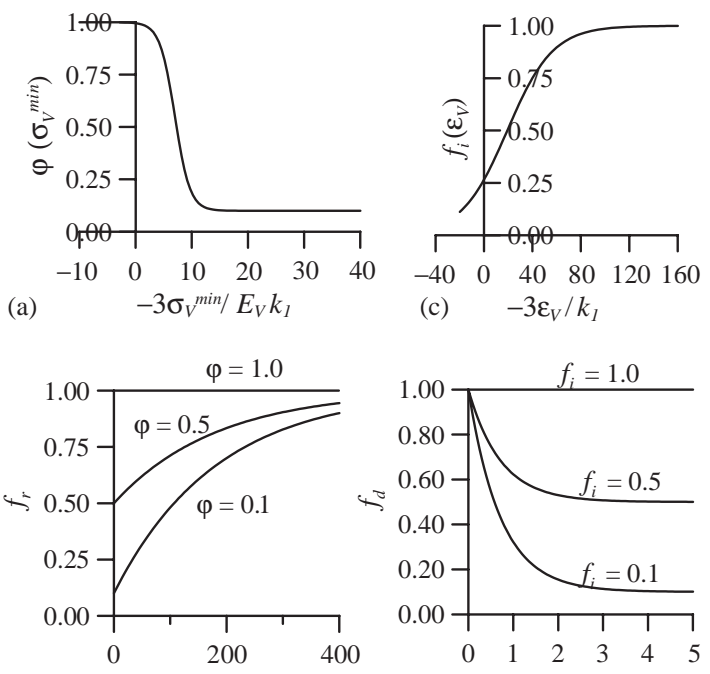

(b)

$$
\left|\varepsilon_{\mathrm{T}} / \mathrm{k}_{1}\right|^{\max }
$$

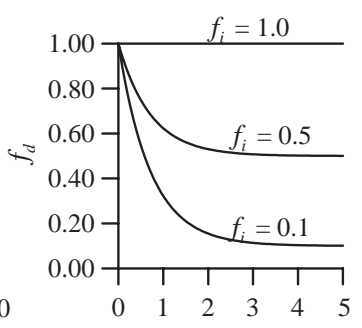

(d) $\quad \mid J_{2}^{0.5 / \varepsilon_{\mathrm{V}} \mid}$

Figure 5. (a) The factor of reduction of the ultimate frictional strength caused by pore collapse; (b) a function smoothing the volumetric boundary; (c) recovery of the reduced shear strength caused by further shear deformation; and (d) the dependence of the volumetric boundary on distortion. 

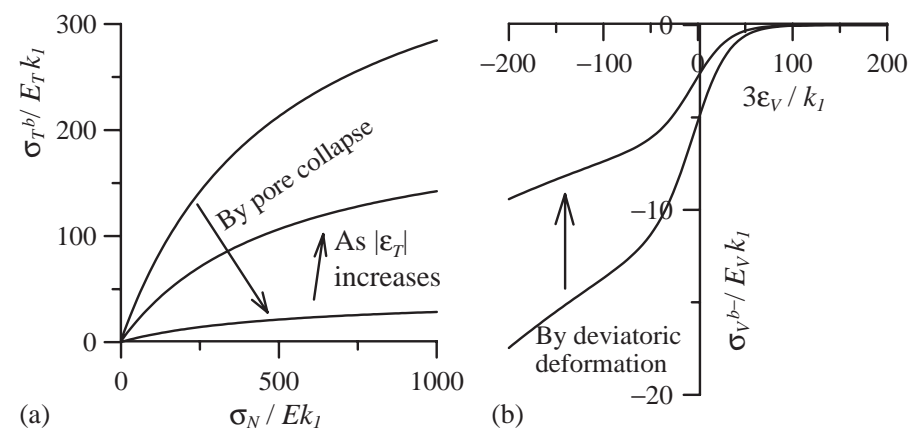

(b)

Figure 6. (a) Dependence of the frictional shear boundary on pore collapse and on shear; and (b) dependence of the volumetric boundary on distortional strain.

While the macroscopic response corresponding to the deviatoric boundaries or the shear boundary is smooth, due to the interaction between many microplanes, the volumetric response is not smooth, according to the boundary definition, because the volumetric stress changes everywhere simultaneously, being common for all the microplanes. The sudden change in the slope of hydrostatic response is partly responsible for the aforementioned excessive lateral contraction in triaxial tests.

Smoothing the volumetric boundary is helpful for reducing the lateral contraction by increasing the slope of the hydrostatic response after pore collapse (Figure 4(b)). It is also physically realistic because not all the pores collapse at the same strain. For this reason, we introduce a smooth signal function $f_{i}$ (Figure 5(c)), which approaches 1 as $\varepsilon_{\mathrm{V}} \rightarrow-\infty$ and 0 as $\varepsilon_{\mathrm{V}} \rightarrow+\infty$;

$$
f_{i}\left(\varepsilon_{\mathrm{V}}\right)=1 /\left[1+\exp \left\{c_{15}\left(3 \varepsilon_{\mathrm{V}} / k_{1}+c_{18}\right)\right\}\right]
$$

where $c_{18}$ is a parameter which describes the centre of the pore collapse transition. Note that (19) uses the same transition centre because it corresponds to the same transition in spite of a different effect.

Under shear strain, rocks, as well as most other geomaterials, exhibit volume changes, either dilatation or compaction [8]. Roughly speaking, porous and soft rocks show shear-enhanced compaction under such a deformation while dense and hard rocks show shear-enhanced dilatation. The shear-enhanced compaction is depicted in Figure 7. Segment $\overline{\mathrm{AB}}$ in the figure represents the deformation under a purely hydrostatic stress increment $\Delta \sigma_{\mathrm{V}}$. Now consider the material at point $\mathrm{B}$ to be subjected to a deviatoric stress increment. Because of the shearenhanced compaction, the response moves from points $\mathrm{B}$ to $\mathrm{C}$ without any change in the hydrostatic stress. As a result, the deviatoric deformation pulls down the volumetric boundary.

Now the question is which variable should be chosen as the measure of the deviatoric distortion. The shear-enhanced compaction is a volume change which is the same on all the microplanes, and so it is not illogical to assume it being governed by a tensorial invariant of strain, for which a natural simple choice is the second deviatoric invariant or the effective deviatoric strain $\bar{e}=\sqrt{J_{2}^{\varepsilon}}$ (which can be regarded as the average of shear strains in all microplanes). It is observed experimentally that the effect gets small at high volumetric strains. Similar to the frictional shear boundary, only the portion of $\varepsilon_{\mathrm{V}}$ due to pore collapse should be affected. So we choose (Figure 5(d)) 


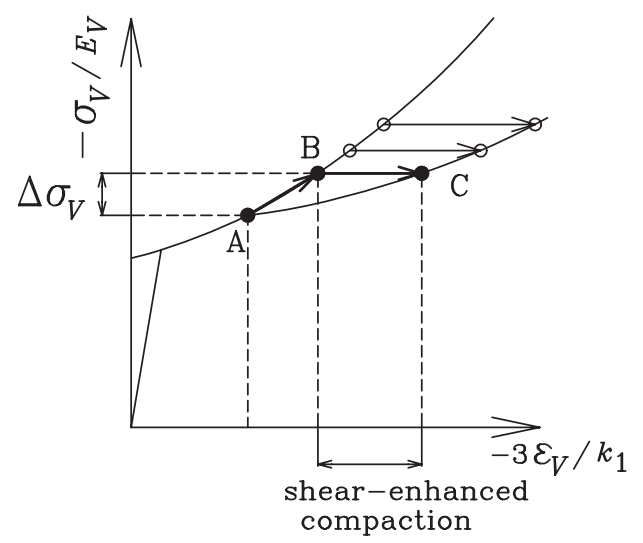

Figure 7. The change of volumetric behaviour caused by shear-enhanced compaction.

$$
f_{d}(\bar{e})=f_{i}\left(\varepsilon_{\mathrm{V}}\right) \exp \left(-c_{24} \bar{e} / \varepsilon_{\mathrm{V}}\right)+\left(1-f_{i}\right)
$$

Based on the foregoing considerations, the final expression for the compressive volumetric boundary for model M4R is

$$
\sigma_{\mathrm{V}}^{b-}=\sigma_{\mathrm{V}}^{b-\mathrm{M} 4} f_{i}\left(\varepsilon_{\mathrm{V}}\right) f_{d}(\bar{e})
$$

which is plotted in Figure 6(b).

Note that the expedient use of tensorial invariant $\bar{e}$ in this equation represents a deviation from the pure microplane modelling concept, in which the microplane stress depends only on the strain components on the same microplane. However, the basic hypothesis of the kinematic constraint is not violated because $\bar{e}$ is an invariant of the same strain tensor as that producing the microplane strains (using a deviatoric stress invariant would be a different matter).

The uniaxial tension introduces significant shear stresses on the microplanes inclined by about $\pm 45^{\circ}$ with respect to the tension direction, and the corresponding microplane shear stress can produce excessively large volume expansion when the shear stresses soften near zero. For this reason, and in view of the experience with model M4 for concrete, it is desirable to introduce a tensile volumetric boundary limiting this expansion:

$$
\sigma_{\mathrm{V}}^{b+}=\frac{E_{\mathrm{V}} k_{1} c_{13}}{\left[1+\left(c_{14} / k_{1}\right)\left\langle\varepsilon_{\mathrm{V}}-k_{1} c_{13}\right\rangle\right]^{2}}
$$

\subsection{Unloading and reloading}

To model unloading, reloading and cyclic loading with hysteresis, we should describe the effect of material damage on the incremental elastic stiffness. The unloading and the reloading may be defined for each microplane strain component separately, which means that some of $\varepsilon_{\mathrm{V}}, \varepsilon_{\mathrm{D}}$, and $\varepsilon_{T}$ may be unloading while at the same time others are loading or reloading. Because work is being recovered from the material during unloading, the unloading occurs in a strain component when the sign of incremental work of the corresponding stress component becomes negative, i.e. $\sigma \Delta \varepsilon<0$. This criterion is considered separately for each microplane, and separately for each microplane strain component. 

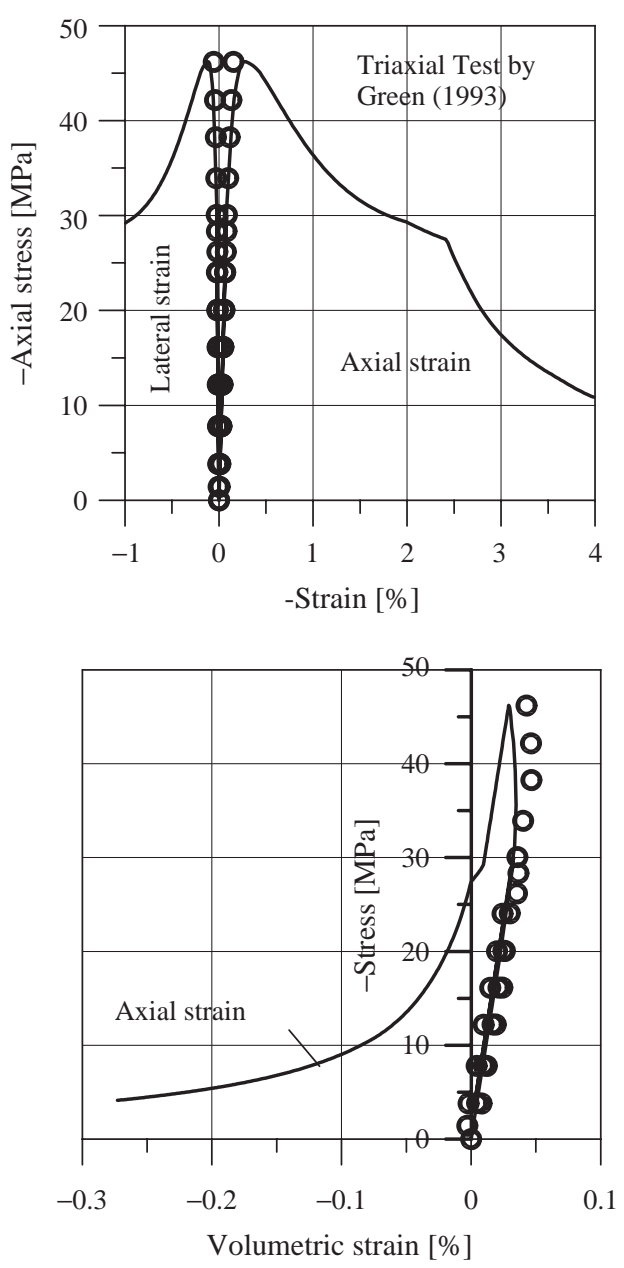

Figure 8. Compressive uniaxial test data (Green 1993) compared to the optimized fit (selected from Green's triaxial test data).

For reloading as well as virgin loading, the incremental moduli are constant and equal to their initial values, $E_{\mathrm{V}}, E_{\mathrm{D}}$ and $E_{T}$, except for compressive volumetric loading. Experiments show that the modulus in such loading conditions never returns to its virgin value $E_{\mathrm{V}}$ but keeps the slope of the hydrostatic boundary for the point at which the slope became higher than the initial slope [27].

The same unloading and reloading rules as used in M4 are adopted here:

$$
E_{\mathrm{V}}^{\mathrm{U}}= \begin{cases}E_{\mathrm{V}}\left(\frac{c_{16}}{c_{16}-\varepsilon_{\mathrm{V}}}+\frac{\sigma_{\mathrm{V}}}{c_{16} c_{17} E_{\mathrm{V}}} \varepsilon_{\mathrm{V}}\right) & \text { for } \varepsilon_{\mathrm{V}} \leqslant 0 \text { and } \sigma_{\mathrm{V}} \leqslant 0 \\ \min \left[\sigma_{\mathrm{V}} / \varepsilon_{\mathrm{V}}, E_{\mathrm{V}}\right] & \text { for } \varepsilon_{\mathrm{V}}>0 \text { and } \sigma_{\mathrm{V}}>0\end{cases}
$$




$$
\begin{aligned}
& E_{\mathrm{D}}^{\mathrm{U}}= \begin{cases}\min \left(E_{\mathrm{D}}\left(1-c_{21}\right)+c_{21} \sigma_{\mathrm{D}} / \varepsilon_{\mathrm{D}}, E_{\mathrm{D}}\right) & \text { for } \sigma_{\mathrm{D}}>0 \text { and } E_{\mathrm{D}} \varepsilon_{\mathrm{D}}>E k_{1} c_{5} \\
\min \left(E_{\mathrm{D}}\left(1-c_{19}\right)+c_{19} \sigma_{\mathrm{D}} / \varepsilon_{\mathrm{D}}, E_{\mathrm{D}}\right) & \text { for } \sigma_{\mathrm{D}}<0 \text { and } E_{\mathrm{D}} \varepsilon_{\mathrm{D}}<-E k_{1} c_{8}\end{cases} \\
& E_{T}^{\mathrm{U}}= \begin{cases}\min \left(E_{T}\left(1-c_{21}\right)+c_{21} \sigma_{T} / \varepsilon_{T}, E_{T}\right) & \text { for } \sigma_{T}>0 \text { and } E_{T} \varepsilon_{T}>E_{T} k_{1} k_{2} \\
\min \left(E_{T}\left(1-c_{19}\right)+c_{19} \sigma_{\mathrm{D}} / \varepsilon_{\mathrm{D}}, E_{\mathrm{D}}\right) & \text { for } \sigma_{T}<0 \text { and } E_{T} \varepsilon_{T}<E_{T} k_{1} k_{2}\end{cases}
\end{aligned}
$$

For more detail regarding other sign combinations of $\varepsilon_{\mathrm{V}}$ and $\sigma_{\mathrm{V}}$, see Reference [27].

\section{EXPLICIT NUMERICAL ALGORITHM TO DELIVER STRESSES FOR GIVEN STRAINS}

In finite-element codes, the global force vector is assembled from the nodal forces obtained by integration of stresses over each element. An explicit constitutive model is needed to calculate the stresses from strains for each integration point of each finite element. The explicit algorithm, similar to that in M4, is as follows:

1. Compute the kinematical transformation matrices $N_{i j}, L_{i j}$ and $M_{i j}$ by which a strain tensor is projected onto each microplane (these matrices are computed only once; the same matrices are used in all strain transformations).

2. At each integration point of each finite element, in each loading step, transform the strain tensor to its vector projections $\varepsilon_{N}, \varepsilon_{L}$ and $\varepsilon_{M}$ on each microplane using $N_{i j}, L_{i j}$ and $M_{i j}$. Check whether each deformation is loading or unloading by means of the unloading criterion $\sigma \Delta \varepsilon<0$, and then compute the associated moduli. Also, obtain the elastic stress increments from (12).

3. After computing $\sqrt{J_{2}^{\varepsilon}}=\sqrt{e_{i j} e_{i j} / 2}, f_{i}$ and $f_{d}$, check whether the computed volumetric and deviatoric stresses satisfy their respective boundaries, i.e. $\sigma_{\mathrm{V}}^{b-} \leqslant \sigma_{\mathrm{V}}^{*} \leqslant \sigma_{\mathrm{V}}^{b+}$ and $\sigma_{\mathrm{D}}^{b-} \leqslant \sigma_{\mathrm{D}} \leqslant$ $\sigma_{\mathrm{D}}^{b+}$. If not, drop them to the boundary values for the given strain.

4. Calculate the average of normal stresses on all the microplanes, $\overline{\sigma_{N}}$, and then recalculate the volumetric stress as $\sigma_{\mathrm{V}}=\min \left(\sigma_{\mathrm{V}}^{*}, \overline{\sigma_{N}}\right)$ where $\sigma_{\mathrm{V}}^{*}$ is the volumetric stress calculated in step 3 (this procedure is essential for obtaining a realistic volume dilatation at low confinement; otherwise, the volumetric stress would unload elastically from the peak stress point in the case of uniaxial compression.

5. Update the record of the minimum volumetric stress $\sigma_{\mathrm{V}}^{\min }$ and the maximum shear strain $\left|\varepsilon_{T}\right|^{\max }$ attained up to now on each microplane. Using these values, compute the shear boundary correction factor $f_{r}$ for each microplane. Check whether the computed shear stresses satisfy the boundaries, $\left|\sigma_{L}\right| \leqslant \sigma_{T}^{b}$ and $\left|\sigma_{M}\right| \leqslant \sigma_{T}^{b}$.

6. Save the strain and stress history for every microplane.

7. Construct the stress tensor for the end of load step from the static equivalence relation, Equation (11) (valid even in the case of volumetric-deviatoric split).

Note that, although the deviatoric stress does not interact with the volumetric stress on the microplane explicitly, it does interact indirectly because the volumetric stress is recalculated in step 4 of the foregoing algorithm. 


\section{DETERMINATION OF MODEL PARAMETERS}

The material parameters of the proposed model are determined by optimum fitting of an extensive set of data from the tests of Salem limestone, sometimes called Indiana or Bedford limestone, conducted at WES [53]. The porosity of this rock is $15.3-16.4 \%$ [53]. The mechanical behaviour of a rock strongly depends on its porosity $[52,54]$. Experiments show that rocks with porosities ranging from $14-35 \%$ behave similarly to concrete, whose porosity is in the same range. The optimized parameters are listed in Table I (the parameters $c_{1}, \ldots, c_{25}$ are all dimensionless, which does not preclude their approximate applicability to other limestones, although calibration tests may be needed). The strength and other basic characteristics of the rock are controlled by four adjustable parameters: $k_{1}$ (which governs radial scaling of the stressstrain curves), $k_{2}$ (ultimate frictional strength), $k_{3}$ (the magnitude of hydrostatic boundary), and

Table I. Parameters of the microplane model proposed.

\begin{tabular}{|c|c|c|}
\hline No. & Description of parameter & Value \\
\hline$E$ & Elastic modulus & $38480 \mathrm{MPa}$ \\
\hline$v$ & Poisson ratio & 0.28 \\
\hline$k_{1}$ & Scales overall stress-strain behaviour radially & $1.43 \times 10^{-4}$ \\
\hline$k_{2}$ & Controls ultimate frictional strength behaviour & $4.30 \times 10^{2}$ \\
\hline$k_{3}$ & Controls pore collapse strength & $1.09 \times 10^{1}$ \\
\hline$k_{4}$ & Controls the hardening rate after the pore collapse & $4.20 \times 10^{2}$ \\
\hline$c_{1}$ & Controls plastic strength of normal boundary & $6.20 \times 10^{-1}$ \\
\hline$c_{2}$ & Controls plastic strain of normal boundary & $2.76 \times 10^{0}$ \\
\hline$c_{3}$ & Controls slope of normal boundary & $4.00 \times 10^{0}$ \\
\hline$c_{4}$ & Scales normal boundary by volumetric strain & $7.00 \times 10^{1}$ \\
\hline$c_{5}$ & Controls plastic strength of positive deviatoric boundary & $1.80 \times 10^{0}$ \\
\hline$c_{6}$ & Controls plastic strain of positive deviatoric boundary & $1.00 \times 10^{0}$ \\
\hline$c_{7}$ & Controls slope of negative deviatoric boundary & $4.00 \times 10^{1}$ \\
\hline$c_{8}$ & Controls plastic strength of negative deviatoric boundary & $3.80 \times 10^{0}$ \\
\hline$c_{9}$ & Controls plastic strain of negative deviatoric boundary & $1.00 \times 10^{0}$ \\
\hline$c_{10}$ & Controls the internal friction angle & $8.40 \times 10^{-1}$ \\
\hline$c_{11}$ & Controls dependence of frictional boundary on confinement & $2.10 \times 10^{0}$ \\
\hline$c_{12}$ & Same as above & $1.00 \times 10^{0}$ \\
\hline$c_{13}$ & Controls plastic strength of positive volumetric boundary & $2.00 \times 10^{-1}$ \\
\hline$c_{14}$ & Controls slope of positive volumetric boundary & $1.00 \times 10^{-1}$ \\
\hline$c_{15}$ & Initial slope of volumetric boundary & $5.29 \times 10^{-2}$ \\
\hline$c_{16}$ & Controls volumetric unloading modulus & $2.00 \times 10^{-2}$ \\
\hline$c_{17}$ & Same as above & $1.00 \times 10^{-2}$ \\
\hline$c_{18}$ & Shift of volumetric boundary & $1.92 \times 10^{1}$ \\
\hline$c_{19}$ & Controls deviatoric unloading modulus & $4.00 \times 10^{-1}$ \\
\hline$c_{20}$ & Controls slope of positive deviatoric boundary & $4.00 \times 10^{1}$ \\
\hline$c_{21}$ & Controls deviatoric unloading modulus & $1.00 \times 10^{0}$ \\
\hline$c_{22}$ & Shear strength reduction by pore collapse & $1.00 \times 10^{-1}$ \\
\hline$c_{23}$ & Slope of pore collapse curve & $2.50 \times 10^{-1}$ \\
\hline$c_{24}$ & Scaling slope of negative volumetric boundary by deformation & $2.38 \times 10^{-1}$ \\
\hline$c_{25}$ & Densification of shear boundary caused by $\left|\varepsilon_{T}\right|^{\max }$ & $5.50 \times 10^{-3}$ \\
\hline
\end{tabular}


$k_{4}$ (the slope of the hydrostatic boundary). In addition, the elastic modulus $E$ controls the vertical scaling of the stress-strain curves (and $k_{1}$ and $E$ can jointly control the horizontal scaling).

It is next to impossible to identify all the parameters by simultaneous optimization at the same time. A successive identification is inevitable [33]. First the elastic modulus $E$ and the Poisson ratio $v$ are easily determined from the initial segments of the axial stress-strain curve and the lateral expansion of uniaxial compression test. From the hydrostatic compression curve, one can then identify the values of products $k_{1} k_{3}, k_{1} k_{4}$, and of $c_{15}$ and $c_{18}$. First, products $k_{1} k_{3}$ and $k_{1} k_{4}$ are determined separately from the response at high pressures, i.e. after pores have collapsed. Then $c_{15}$ and $c_{18}$ are determined from the initial segment of the hydrostatic curve, in which the pores are still collapsing. Parameter $c_{25}$ is identified by matching the shear-enhanced compaction at high confinement.

The values of products $k_{1} c_{5}, k_{1} c_{20}, k_{1} c_{8}$ and $k_{1} c_{7}$, and of $c_{6}$ and $c_{9}$ are obtained from the postpeak response in uniaxial compression or tension, or from triaxial tests at low confinement. The roundness of the peaks of the stress-strain curves is adjusted by varying the length of the horizontal boundaries (yield limits), characterized by $c_{6}$ and $c_{9}$. The values of $k_{1} c_{7}$ and $k_{1} c_{20}$ are then determined from the post peak slopes. The values of $k_{1} c_{5}$ and $k_{1} c_{8}$ are determined from the lateral expansion under compressive loading. The ratio of the negative deviatoric boundary to the positive one is figured out from the dilatation at low confinement (when $\left|\sigma_{\mathrm{D}}^{b-}\right| / \sigma_{\mathrm{D}}^{b+}>1$, the material expands; otherwise, it contracts).

Using triaxial test data, one can further figure out the parameters associated with the shear boundary. Parameter $c_{10}$ and product $k_{1} c_{11}$ are determined from the internal friction angle and the cohesion at zero normal stress. The cohesion reduction factor given by the ratio $c_{12} / k_{1}$ could be determined from combined shear and tension test data but the same value as identified for concrete in model M4 is used because such data are not available. The value of product $k_{1} k_{2}$ can be ascertained from the change of the friction angle at high pressures. Based on the lateral deformation in triaxial tests, one can obtain parameters $c_{18}, c_{22}$ and $c_{23}$ which characterize the shear strength reduction caused by pore collapse. Based on the gradual strength gain at high pressures, one can find the value of ratio $c_{26} / k_{1}$.

Note that $k_{1}$ is an extra parameter that cannot be uniquely determined from test data. It is nevertheless convenient to use $k_{1}$ for simultaneous scaling of all the $c$-parameters. Therefore, the $c$-parameters need to be determined only after $k_{1}$ has been chosen.

The remaining parameters, which characterize the normal boundary and the tensile volumetric boundary, are associated with tensile behaviour. Because tensile data are not available, the values previously identified for model M4 are retained.

\section{CALIBRATION AND COMPARISON WITH TEST DATA}

The fits of test data shown in Figures 9-11 are all obtained with one and the same set of material parameters, listed in Table I. The strength in uniaxial compression is matched closely (Figure 8). The post-peak response cannot be compared because it was not measured in these experiments. However, the simulations agree with the typical post-peak response, especially the volumetric expansion, generally expected for quasibrittle geomaterials. The volumetric contraction before the peak is matched well. At large post-peak compressive strains, the axial stress should get reduced to zero, and that is what the model furnishes. 


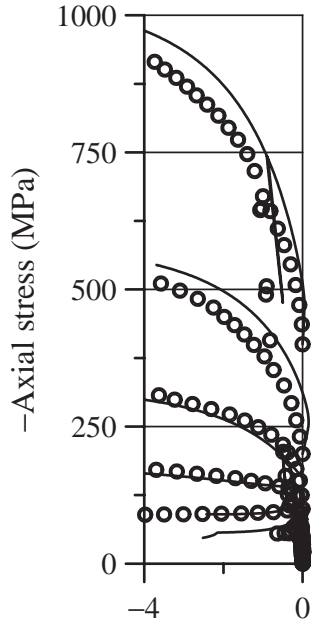

(a)

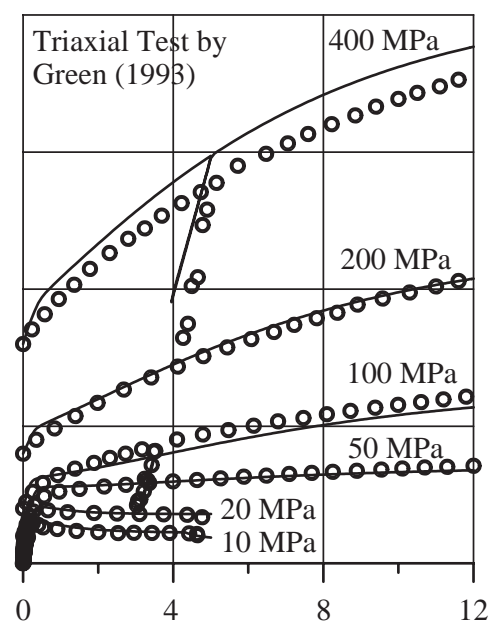

- Strain $(\%)$

Figure 9. Standard triaxial test data [53] compared to the optimized fit; (a) lateral strain and (b) vertical strain.

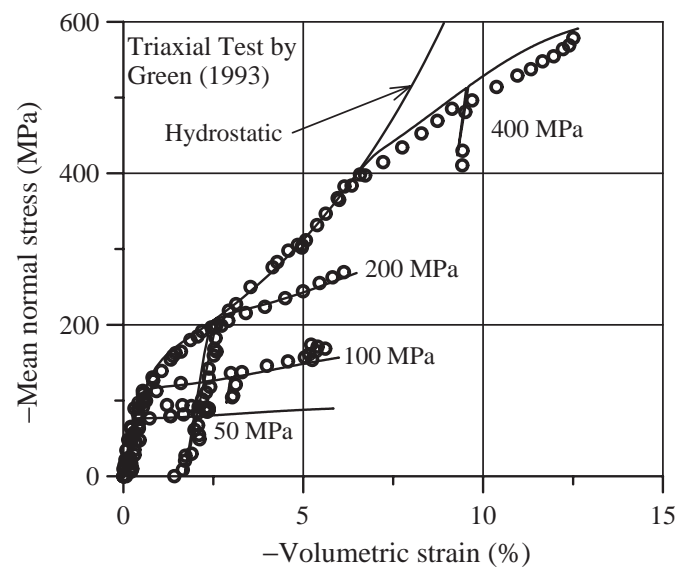

Figure 10. Hydrostatic (volumetric) pressure test data [53] compared to the optimized fit (the stress and strain are compressive).

Figure 9 depicts the fits of triaxial test data at confinement pressures 10, 20, 50, 100, 200 and $400 \mathrm{MPa}$. The rock is seen to become rather ductile under confining pressures higher than $50 \mathrm{MPa}$. This brittle-ductile transition is reproduced properly. The sudden drop of the macroscopic deviatoric stiffness, the gradual recovery without excessive lateral contraction, and the gradual strength gain at very high pressures, exhibited by experiments, are well captured by the model. The fitting is carried out through the full strain range, up to strain $12 \%$. Good test data simulation is thus demonstrated. 

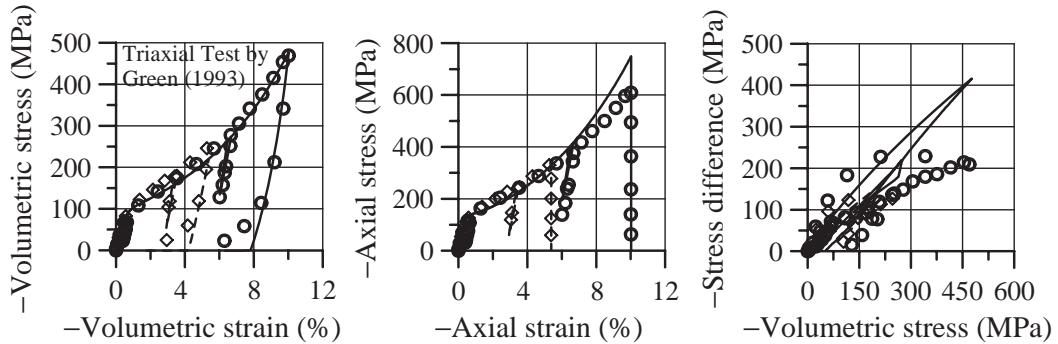

Figure 11. Uniaxial strain test data [53] compared to the optimized fit.

The measured hydrostatic response is fitted in Figure 10, and so are the volume changes in triaxial tests. Because of the dilatation due to the distortion (shearing), the response stress-strain curve deviates from the hydrostatic curve as soon as the stress difference becomes non-zero.

Figure 11 presents the fitting of the uniaxial strain tests, i.e. confined compression tests at zero lateral strain. Difficulties with data fitting arose in this case. In the test, first, the specimen is loaded axially while the lateral strain is kept zero (at the gauge by which it is measured). Then it is unloaded laterally while the vertical strain is kept constant. This means that the lateral strain is zero only at the gauge while the average lateral strain over the specimen length need not be exactly zero. This fact may explain why the fits are not too good. However, they are not poor either, except for the volumetric stress as a function of the stress difference.

\section{LOCALIZATION LIMITERS}

In view of strain softening, applications in finite-element programs must give proper attention to localization of damage and avoidance of spurious mesh sensitivity. After two decades of research, these aspects are now relatively well understood [59] and need not be discussed here in detail.

In problems of impact, which have been the main motivation of this study, spurious localization is temporarily prevented (and often delayed beyond the duration of interest) by two measures: (1) viscous damping, which is always present in the explicit finite-element model, and (2) inertia effects, which prevent localization during the extremely short duration of an impact event.

In problems of longer duration, there are several possible localization limiters $[35,36]$ to be used with the present model: (1) the simple crack band model, in which the element size is restricted to be roughly the same as the size of the test specimens by which the model has been calibrated, which is about $50 \mathrm{~mm}$ in the present case; or (2) the general crack band model, in which the element size may be changed if a certain particular adjustment of the post-peak softening is made, either (a) on the microplane level, as implemented in model M5 [40] developed in parallel, or (b) on the element level, by introducing localization element consisting of softening and unloading overlays $[55,56]$. 
Another, more general, localization limiter is the integral-type non-local approach. A method to combine non-locality with the microplane model has been suggested in References $[31,32,57]$ and developed, with numerical demonstrations, in Reference [58]. Gradient-type localization limiters could of course be introduced as well.

\section{CONCLUSION}

The paper deals with contiguous rock located between rock joints. Based on microplane model M4 for concrete adopted as the point of departure, a kinematically constrained microplane constitutive model for hardening and softening non-linear triaxial behaviour of isotropic porous rock has been developed and calibrated by extensive test data for limestone. Except for a small iterative adjustment of pressure sensitivity of some microplane coefficients, the model yields the stress tensor from the given strain tensor explicitly, which is suitable for explicit finite-element programs.

Thanks to the microplane framework, in which the constitutive relation is expressed in terms of stress and strain vectors rather than tensors, the new model has some important advantages over the classical constitutive models for rock expressed in terms of the stress and strain tensors and their invariants. They include:

(1) conceptual simplicity;

(2) decoupling of the constitutive modelling from the tensorial invariance restrictions, which are satisfied automatically;

(3) direct simulation of oriented physical mechanisms associated with internal surfaces, such as tensile cracking of a particular orientation, slip and friction, dilatancy due to slip in a particular direction, and lateral expansion due to compression splitting of a particular direction;

(4) automatic representation of the vertex effect, made possible by the existence of numerous simultaneous yield surfaces in the microplane system;

(5) automatic simulation of cross effects such as dilatancy due to shear, made possible by the kinematic constraint;

(6) complex path-dependence generated by numerous possible combinations of loading and unloading on various microplanes;

(7) simulation of the accumulation of residual stresses in the microstructure due to fatigue loading;

(8) automatic representation of progressive hardening, due to the fact that different microplanes enter yielding at different times;

(9) ease of possible generalization to orthotropy; and

(10) easy implementation of strain-softening yield limits (which would be forbiddingly complex on the tensorial level).

Compared to model M4, the following innovations and modifications are made: (1) The effect of pore collapse is modelled more realistically. (2) Excessive lateral contraction in triaxial tests is eliminated, by virtue of simulating the reduction of frictional strength caused by pore collapse. (3) The recovery of frictional strength in shear deformation is captured, which allows representation of the gradual hardening of the material at increasing confining pressures. 
(4) The shear-enhanced compaction in triaxial tests, which is manifested by a deviation from the hydrostatic stress strain curve, is modelled, which is achieved by a dependence of the volumetric boundary on the strain intensity, $\sqrt{J_{2}^{\varepsilon}}$.

\section{ACKNOWLEDGEMENTS}

Grateful appreciation is due to the U.S. Army Engineer Waterways Experiment Station (WES), Vicksburg, Mississippi, for funding this work under Contract DACA39-94-C-0025 with Northwestern University.

\section{REFERENCES}

1. Costin LS. A microcrack model for the deformation and failure of brittle rock. Journal of Geophysical Research 1985; 88:9485-9492.

2. Zhao Y. Crack pattern evolution and a fractal damage constitutive model for rock. International Journal of Rock Mechanics and Mining Sciences 1998; 35(3):349-366.

3. Homand F, Hoxha D, Belem T, Pons MN, Hotetit N. Geometric analysis of damaged microcracking in granites. Mechanics of Materials 2000; 32:361-376.

4. Hoxha D, Homand F. Microstructural approach in damage modelling. Mechanics of Materials 2000; 32:377-387.

5. Shao JF, Rudnicki JW. A microcrack-based continuous damage model for brittle geomaterials. Mechanics of Materials 2000; 32:607-619.

6. Pan YW, Wen BH. Constitutive model for the continuous damage of brittle rock. Géotechnique 2001; 51(2):155-159.

7. Ortiz M. A constitutive theory for the inelastic behaviour of concrete. Mechanics of Materials 1985; 4:67-93.

8. Ofoegbu GI, Curran JH. Deformability of intact rock. International Journal of Rock Mechanics and Mining Sciences and Geomechanics Abstracts 1992; 29(1):35-48.

9. Taylor GI. Plastic strain in metals. The Journal of the Institute of Metals 1938; 62:307-324.

10. Batdorf SB, Budianski B. A mathematical theory of plasticity based on the concept of slip. Technical Note No. 1871, National Advisory Committee for Aeronautics: Washington, DC, 1949.

11. Kröner E. Zur Plastischen Verformung des Vielkristalls. Acta Metallurgica 1961; 9:155-161.

12. Budiansky B, Wu TT. Theoretical prediction of plastic strains of polycrystals. In Proceedings of 4th U.S. National Congress of Applied Mechanics. ASME: New York, 1962; 1175-1185.

13. Lin TH, Ito $\mathrm{M}$. Theoretical plastic distortion of a polycrystalline aggregate under combined and reversed stress. Journal of Mechanics and Physics of Solids 1965; 13:103-115.

14. Hill R. Continnuum micromechanics of elastoplastic polycrystals. Journal of Mechanics and Physics of Solids 1965; 13:89-101.

15. Hill R. Generalized constitutive relations for incremental deformations of metal crystals by multi-slip. Journal of Mechanics and Physics of Solids 1966; 14:95-102.

16. Rice JR. On the structure of stress-strain relations for time-dependent plastic deformation of metals. Journal of Applied Mechanics ASME 1970; 37:728-737.

17. Zienkiewicz OC, Pande GN. Time-dependent multi-laminate model of rocks-A numerical study of deformation and failure of rock masses. International Journal of Numerical and Analytical Methods in Geomechanics 1977; 1: 219-247.

18. Pande GN, Sharma KG. Implementation of computer procedures and stress-strain laws in geotechnical engineering. In Proceedings of Symposium on Implementation of Computer Procedures and Stress-Strain Laws in Geotechnology Engineering, Desai CS, Saxena SK (eds). Acorn Press: Durham, NC, 1981; 575-590.

19. Pande GN, Sharma KG. Multi-laminate model of clays-a numerical evaluation of the influence of rotation of the principal stress axes. Journal of Engineering Mechanics ASCE 1983; 109(7):397-418.

20. Pande GN, Xiong W. An improved multi-laminate model of jointed rock masses. In Proceedings of the First International Symposium on Numerical Models in Geomechanics, Balkema: Rotterdam, the Netherlands, 1982; 218 226.

21. Bažant ZP. Microplane model for strain controlled inelastic behaviour. In Mechanics of Engineering Materials, Desai CS, Gallagher RH (eds), Chapter 3. Wiley: London, 1984; 45-59.

22. Bažant ZP, Oh BH. Microplane model for progressive fracture of concrete and rock. Journal of Engineering Mechanics ASCE 1985; 111:559-582.

23. Bažant ZP. Instability, ductility, and size effect in strain-softening concrete. Journal of Engineering Mechanics ASCE 1976; 102(EM2):331-344 (disc. 103:357-358, 775-777, 104:501-502).

24. Bažant ZP, Oh BH. Crack band theory for fracture of concrete. Materials and Structures 1983; 16:155-177. 
25. Brocca M, Bažant ZP. Microplane model and metal plasticity. Applied Mechanics Reviews ASME 2000; 53(10): $265-281$.

26. Bažant ZP, Prat PC. Microplane model for brittle plastic material: I. Theory. Journal of Engineering Mechanics ASCE 1988; 114:1672-1688.

27. Bažant ZP, Caner FC, Adley MD, Akers SA. Microplane model M4 for moncrete I: formulation with workconjugate deviatoric stress. Journal of Engineering Mechanics ASCE 2000; 126(9):944-953.

28. Carol I, Bažant ZP. Damage and plasticity in microplane theory. International Journal of Solids and Structures 1997; 34(29):3807-3835.

29. Carol I, Jirásek M, Bažant ZP. A thermodynamically consistent approach to microplane theory: Part I. Free energy and consistent microplane stresses. International Journal of Solids and Structures 2001; 38:2921-2931.

30. Bažant ZP, Prat PC. Microplane model for brittle plastic material: II. Verification. Journal of Engineering Mechanics ASCE 1988; 114:1689-1702.

31. Bažant ZP, Xiang Y, Prat PC. Microplane model for concrete. I. Stress-strain boundaries and finite strain. Journal of Engineering Mechanics ASCE 1996; 122(3):245-254 (with Errata 123(3):411).

32. Bažant ZP, Xiang Y, Adley MD, Prat PC, Akers SA. Microplane model for concrete: II. Data delocalization and verification. Journal of Engineering Mechanics ASCE 1996; 122(3):255-262.

33. Caner FC, Bažant ZP. Microplane Model M4 for Concrete II: algorithm and calibration. Journal of Engineering Mechanics ASCE 2000; 126(9): 954-961.

34. Brocca M, Bažant ZP. Microplane finite element analysis of tube-squash test of concrete with shear angles up to $70^{\circ}$. International Journal for Numerical Methods in Engineering 2001; 52:1165-1188.

35. Bažant ZP, Planas J. Fracture and Size Effect: in Concrete and Other Quasibrittle Materials. CRC Press: New York, 1998.

36. Jirásek M, Bažant ZP. Inelastic Analysis of Structures. Wiley: New York, 2002.

37. Bažant ZP, Caner FC, Adley MD, Akers SA. Fracture rate effect and creep in microplane model for dynamics. Journal of Engineering Mechanics ASCE 2000; 126(9):955-970.

38. Caner FC, Bažant ZP, Červenka J. Vertex effect in strain-softening concrete at rotating principal axes. Journal of Engineering Mechanics ASCE 2002; 128(1):24-33.

39. Ožbolt J, Bažant ZP. Microplane model for cyclic triaxial behaviour of concrete. Journal of Engineering Mechanics ASCE 1992; 118(7):1365-1386.

40. Bažant ZP, Zi G, Jendele L. Microplane model for quasibrittle materials based on fracture energy of cohesive crack. 2002 ; in preparation.

41. Bažant ZP, Prat PC. Creep of anisotropic clay: new microplane model. Journal of Engineering Mechanics ASCE 1987; 113(7):1000-1064.

42. Prat PC, Bažant ZP. Microplane model for triaxial deformation of saturated cohesive soils. Journal of Geotechnical Engineering ASCE 1991; 117(6):891-912.

43. Brocca M, Brinson C, Bažant ZP. Three-dimensional constitutive model for shape memory alloys based on microplane model. Journal of the Mechanics and Physics of Solids 2002; 50: 1051-1077.

44. Brocca M, Bažant ZP, Daniel IM. Microplane model for stiff foams and finite element analysis of sandwich failure by core indentation. International Journal of Solids and Structures 2001; 38:8111-8132.

45. Bažant ZP, Oh BH. Efficient numerical integration on the surface of a sphere. Zeitschrift für angewandte Mathematik und Mechanik (ZAMM, Berlin) 1986; 66(1):37-49.

46. Carol I, Bažant ZP, Prat PC. Geometric damage tensor based on microplane model. Journal of Engineering Mechanics 1991; 117(10):2429-2448.

47. Bažant ZP, Cedolin L. Stability of Structures: Elastic, Inelastic, Fracture, and Damage Theories. Oxford University Press: New York, 1991.

48. Jirásek M. Modeling of fracture and damage in quasibrittle materials. Ph.D. Dissertation, Northwestern University: Evanston, IL, 1993.

49. Ožbolt J, Li Y, Kožar I. Microplane model for concrete with relaxed kinematic constraint. International Journal of Solids and Structures 2001; 38:2683-2711.

50. Bažant ZP, Gambarova P. Crack shear in concrete: crack band microplane model. Journal of Structural Engineering ASCE 1984; 110:2015-2035.

51. Sfer D, Carol I, Gettu R, Etse G. Experimental study of the triaxial behaviour of concrete. Report GT021/2000, ETECCPB (Sch. of C. E., UPC): Barcelona, Spain, 2000.

52. Wong TF, Baud P. Mechanical Compaction of porous sandstone. Oil \& Gas Science and Technology-Rev. IFP 1999; 54(6):715-727.

53. Green ML. Mechanical response of SRII Salem Limestone. Report CEWES-SD-0 (70-1r). Waterways Experiment Station, 1993.

54. Baud P, Schubnel A, Wong TF. Dilatancy, compaction, and failure mode in Solnhofen limestone. Journal of Geophysical Research 1999; 105(B8):19289-19303.

55. Bažant ZP, Červenka J, Wierer M. Equivalent localization element for crack band model and as alternative to elements with embedded discontinuities. In Fracture Mechanics of Concrete Structures (Proceedings of FraMCoS-4 
International Conference Paris), de Borst R, Mazars J, Pijaudier-Cabot G, van Mier JGM (eds). Swets \& Zeitlinger (A.A. Balkema Publishers): Lisse, 2001; 765-772.

56. Cervenka J, Bažant ZP, Wierer M. Equivalent localization element for crack band approach to mesh-size sensitivity in microplane model. International Journal for Numerical Methods in Engineering 2002; submitted.

57. Bažant ZP, Ožbolt J. Nonlocal microplane model for fracture, damage, size effect in structures. Journal of Engineering Mechanics ASCE 1990; 116(11):2484-2504.

58. di Luzio G, Bažant ZP, Cedolin L. Nonlocal generalization of microplane model. Report, Northwestern University. International Journal of Solids and Structures, 2002; to be submitted.

59. Bažant ZP, Jirásek M. Nonlocal integral formulations of plasticity and damage: survey of progress. Journal of Engineering Mechanics ASCE 2002; 128(11): 1119-1149. 